\title{
Small bowel imaging in Crohn's disease patients
}

\author{
James Gauci ${ }^{a}$, Lara Sammut ${ }^{b}$, Martina Sciberras ${ }^{a}$, Naomi Piscopo ${ }^{a}$, Kristian Micallef ${ }^{b}$, Kelvin Cortis ${ }^{b}$, \\ Pierre Ellula
}

Mater Dei Hospital, Malta

\section{Abstract}

\section{Introduction}

Crohn's disease $(\mathrm{CD})$ is a lifelong, chronic inflammatory bowel disease (IBD), with a relapsing and remitting nature. The small bowel (SB) is involved to a varying extent in more than two thirds of CD patients. It can run an inflammatory course or a more complicated one with stricture, fistula, and abscess formation [1].

Esophagogastroduodenoscopy (EGD) and ileocolonoscopy with biopsies are the conventional endoscopic techniques that usually enable a diagnosis of IBD. While the duodenal and ileocecal region are usually visualized adequately with these techniques, this may not be the case with other SB segments, because of either their deep location or stricture formation. It has been shown that examination can miss terminal ileal $C D$,

${ }^{a}$ Division of Gastroenterology (James Gauci, Martina Sciberras, Naomi Piscopo, Pierre Ellul); ${ }^{b}$ Department of Medical Imaging (Lara Sammut, Kristian Micallef, Kelvin Cortis), Mater Dei Hospital, Malta

Conflict of Interest: None

Correspondence to: Dr. Pierre Ellul, $2^{\text {nd }}$ Floor, Brown Block Mater Dei Hospital, Msida, MSD 2090, Malta e-mail: ellul.pierre@gmail.com

Received 19 October 2017; accepted 27 February 2018; published online 4 May 2018

DOI: https://doi.org/10.20524/aog.2018.0268 as the latter can skip the distal ileum or involve the submucosal layers and the mesentery [2].

There are various modalities that allow for further assessment of the SB, including radiologic procedurescomputed tomographic enterography (CTE), magnetic resonance enterography (MRE) and ultrasonography (US) and other endoscopic techniques (capsule endoscopy [CE] and enteroscopy). These modalities assist conventional endoscopy in establishing the diagnosis of $\mathrm{CD}$, as well as determining the disease extent, activity and response to therapy. Moreover, radiologic modalities allow for the appreciation of extraintestinal complications. The aim of this review is to analyze the various modalities currently available.

\section{Cross-sectional imaging techniques}

Cross sectional imaging techniques: technical issues

\section{Computed tomography (CT)}

Conventional abdominopelvic CT has traditionally been used to assess intestinal obstruction and extraintestinal complications of $\mathrm{CD}$, such as abscesses and fistulas. It has a minimal role in the identification of SB $\mathrm{CD}$ [3] because 
of difficulty with the interpretation of collapsed bowel loops, which can hide lesions or simulate pathological wall thickening [4].

CTE and enteroclysis improve visualization of the SB mucosa and wall as they allow for bowel distension, a fundamental requisite for SB imaging [5]. Luminal distension can be achieved with enteric contrast agents, classified as neutral or positive, depending on their X-ray attenuation.

In CTE, contrast is given orally; 1-2 L are usually ingested 30$45 \mathrm{~min}$ before the procedure [5]. Neutral contrast agents have $\mathrm{X}$-ray attenuation similar to water. To minimize absorption, water is usually mixed with high molecular size compounds that do not alter water density, such as polyethylene glycol, mannitol, sugar alcohols or sorbitol. Positive contrast agents are usually a mixture of barium sulfate (1-2\%) or iodinated contrast agents (2-3\%), and are preferred for the evaluation of perforations or fistulas in CD [6]. CT enteroclysis necessitates the placement of a nasojejunal tube under fluoroscopic guidance for enteric contrast administration.

The use of intravenous contrast agents, with imaging after intravenous contrast administration, is mandatory with these techniques. The presence of different contrast enhancement patterns of the bowel wall and the mesenteric vessels will facilitate the assessment of disease activity [7].

CTE has been shown to positively influence the management of patient care with $\mathrm{CD}$ [8]. It can assist the diagnosis of SB $\mathrm{CD}$, determine the degree of inflammation, and assess for complications and extraintestinal features.

\section{Magnetic resonance imaging (MRI)}

MRE has become an increasingly important imaging modality in the initial assessment of SB CD, as well as in the monitoring of disease activity [9]. MRI can also help in the assessment of perianal disease but this requires a dedicated examination of the pelvis with appropriate high resolution sequences (predominantly short T1 inversion recovery sequences) and is not included in the published/widely used MRE protocols [9].

Acutely inflamed SB segments in CD patients demonstrate increased perfusion and restricted diffusion. Diffusionweighted imaging (DWI) and dynamic contrast enhanced MRI sequences are both promising techniques for the detection of active SB inflammation and provide quantitative measures of bowel perfusion and diffusion that can differentiate actively inflamed SB segments from normal SB in CD $[9,10]$.

In MRE, oral contrast agents are classified according to the action on the signal intensity of bowel lumen: positive (currently not used), negative and biphasic agents. Negative contrast agents consist of a super-paramagnetic nonabsorbable solution of iron oxide particles. These reduce the signal of the intestinal lumen, on both T1- and T2-weighted images, producing a "black lumen" effect [6]. Biphasic contrast agents are water-based agents that contain non-absorbable additives to create a hyperosmolar solution (polyethylene glycol, sorbitol, lactulose, mannitol solutions) that produces a negative effect on T1-weighted and a positive effect on T2weighted images. This is referred to as a "water-like" effect [6].
When oral contrast is used, a total volume of $1.5 \mathrm{~L}$ is usually ingested, and image acquisition should be performed 40-60 min after the ingestion in order to achieve maximal bowel distension [11]. DWI MRE without intravenous contrast material (gadolinium) was found to be non-inferior to contrastenhanced MRE in the evaluation of SB inflammation in CD [12].

MRE has been shown to alter the management of patients with ileocolonic CD. In a study where patients with ileocolonic CD underwent routine MRE, 53\% had additional medical management for previously non-diagnosed active inflammation, while $16 \%$ underwent surgery for complicated $\mathrm{CD}$ or medical intractability. In all surgical patients, the intraoperative findings were consistent with the MRE diagnosis, demonstrating the reliability of this technique [13].

Another similar study demonstrated that MRE influenced a change in treatment in $55.3 \%$ of known CD patients. This included starting immunosuppressants (10.7\%), starting or switching anti-tumor necrosis factor- $\alpha$ drugs (27.3\%), surgical intervention (10\%) and de-escalation in treatment from combined immunosuppressive therapy to monotherapy (2\%) [14]. Similarly to CT enteroclysis, MR enteroclysis can also be performed after inserting a nasojejunal tube under radiological guidance.

\section{US}

US enables the rapid evaluation of bowel wall thickness and stratification of the bowel located in the right iliac fossa. For a comprehensive entire SB evaluation, the examination time is much longer. Moreover, oral contrast should be used in stateof-the-art whole SB assessment with US [15].

Contrast-enhanced US has been shown to be a useful addition in imaging IBD patients as it can determine disease activity in $\mathrm{CD}$ patients with more precision [15]. Intravenous microbubble contrast agents can be used in combination with low mechanical index imaging modes or power Doppler. This will enable better visualization of the vascularity within the thickened bowel walls $(>3 \mathrm{~mm}$ ), assessment of mesenteric lymph nodes, and differentiation between inflammatory and fibrotic strictures [16].

Hence, US also plays an important role in the management of CD patients. One of the drawbacks of US is its reliance on operator experience, not widely available [17].

\section{Cross sectional imaging techniques: findings}

\section{Inflammatory activity}

The radiological features of underlying active inflammatory $\mathrm{CD}$ are presented in Table 1 . Bowel-wall thickening $>3 \mathrm{~mm}$ is defined as abnormal, with thickening $>6 \mathrm{~mm}$ being classified as severe. Segmental mural hyperenhancement of SB loops relative to normal loops (Fig. 1A,B) correlate significantly with the histological finding of active CD $[18,19]$. Increased bowel wall vascularization on color Doppler interrogation or contrast-enhanced US is always a sign of active inflammation 
and has been significantly related to the clinical activity indices [20] and endoscopic activity [21,22] in CD.

Additional signs of mural disease may include ulceration (Fig. 1C) and a "cobblestone" appearance, characteristic of multiple continuous bowel wall ulcerations. Mural stratification entails the visualization of the different layers of the bowel wall (Fig. 1A). When present, the inner mucosa and outer serosa enhance avidly, but the intervening bowel wall can have various degrees of attenuation, depending on what pathological process is present. The presence of intramural edema (target sign) indicates active inflammation, whilst the presence of intramural fat indicates past or chronic inflammation on CTE and MRE [18]. On US, partial or complete loss of the normal gut stratification results in diffuse hypoechogenicity of the bowel wall with a central echogenic line corresponding to the narrowed bowel [23].

Table 1 Radiological features of inflammatory activity

\begin{tabular}{l} 
Bowel wall thickening \\
Bowel wall hyperenhancement \\
Bowel wall ulceration (cobble stone appearance) \\
Intramural edema (target sign) on mural stratification \\
Mesenteric fibrofatty proliferation (creeping fat phenomenon) \\
Mesenteric hypervascularity (comb sign) \\
Enlarged mesenteric lymph nodes \\
Intestinal stenosis \\
\hline
\end{tabular}
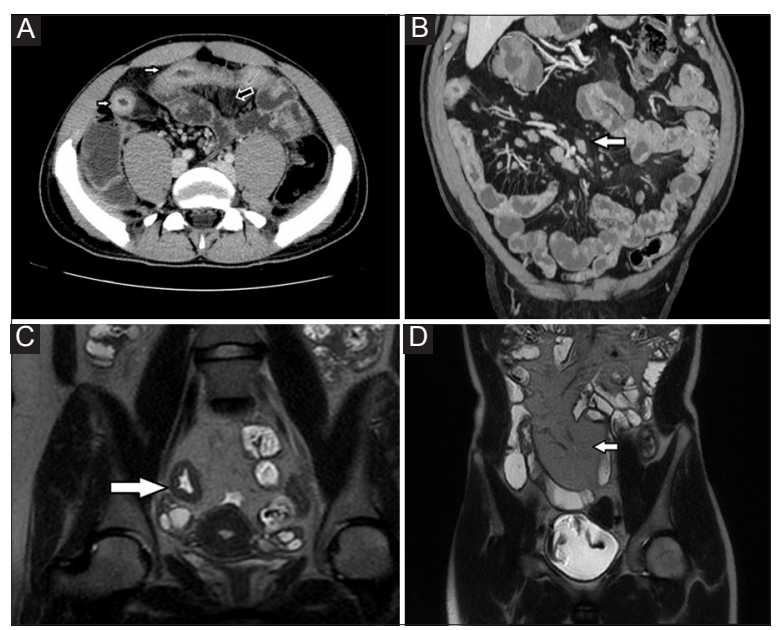

Figure 1 (A) Axial computed tomography (CT) enterogram section shows mucosal hyperenhancement and mural stratification of the actively inflamed distal ileal loops (white arrows), as well as mesenteric hypervascularity adjacent to the involved bowel segment, known as the "comb sign" (black arrow); (B) Coronal CT enterogram section shows multiple enlarged mesenteric lymph nodes (arrow) as well as mesenteric hypervascularity and increased enhancement of the distal ileal loops in keeping with ongoing inflammation; (C) Coronal T2weighted single-shot fast spin-echo image shows a mural ulcer in an inflamed segment of distal ileum (arrow); (D) Coronal T2-weighted single-shot fast spin-echo image of the abdomen shows fibrofatty proliferation in the right iliac fossa adjacent to a chronically inflamed terminal ileum (arrow), also known as the "creeping fat phenomenon"
A distinct advantage of cross-sectional imaging compared to endoscopy is its ability to evaluate the mesentery. CT density of the mesentery $>60$ Hounsfield units (HU) is usually defined as severe disease, levels $>20 \mathrm{HU}$ but $\leq 60 \mathrm{HU}$ are classified as mild, and levels $\leq 20 \mathrm{HU}$ are considered normal [24]. Mesenteric fibrofatty proliferation is seen as finger-like projections of mesenteric fat, which extend towards and encase the anti-mesenteric border of the bowel, resulting in bowel loop separation [23]. Although this "creeping fat phenomenon" (Fig. 1D) is a sign of CD, it persists in clinically quiescent disease [18].

The severity of mesenteric hypervascularity, known as the "comb sign" (Fig. 1A,B), is determined according to the diameter of each vessel. Vessels exhibiting a diameter twice that of normal are usually considered severe, while those with a diameter less than twice the normal diameter, but still with noticeable expansion, are considered mild. This is created by edematous, engorged and hypervascular mesenteric vasa recta vessels, which penetrate the bowel wall perpendicular to the bowel lumen [18]. Engorged vasa recta indicate active inflammation and have been associated with elevated C-reactive protein (CRP) levels and longer hospital stays in patients with severe $\mathrm{CD}$ [25].

The presence of enlarged mesenteric lymph nodes (>10 mm) is another feature of active inflammation (Fig. 1B). Intestinal stenosis is classified according to the constitutive narrowness of the intestinal lumen [26]. A lumen $>11 \mathrm{~mm}$ is defined as normal, $<6 \mathrm{~mm}$ as severe, with measurements in between 6 and $11 \mathrm{~mm}$ being classified as mild [27].

A systematic review comparing MRE and CTE in the evaluation of SB CD demonstrated their role in the correct identification of disease activity. The pooled sensitivity for detecting active SB CD was $87.9 \%$ for MRE and $85.6 \%$ for CTE. The pooled specificity was $81.2 \%$ for MRE and $83.6 \%$ for CTE [28]. Another study by Paquet et al demonstrated that the radiological features of wall thickness, mesenteric fat stranding, mesenteric lymphadenopathy and mesenteric abscess positively correlated with the histological inflammatory activity score [29].

\section{Fistulas}

Fistulas usually originate from the serosal surface of bowel loops that exhibit signs of active inflammation and connect the diseased bowel to another organ, another bowel segment, or the skin surface. They generally appear as hyperenhancing tracts on CTE and as linear regions of T2 hyperintense signal on MRE. One exception is a perianal fistula (Fig. 2A), often isoattenuating relative to the anorectum, potentially because of its chronicity [18].

CT and MRI are the preferred imaging tools for the detection of intra-abdominal fistulas [1]. An enteroenteric fistula may appear as an enhanced sinus tract between adjacent bowel loops. A "star sign" (Fig. 2B) represents a conglomerate of inflamed bowel loops interconnected by multiple fistulous tracts. Detection of enterovesical (Fig. 2C) and enterovaginal fistulas can be challenging. This may however be facilitated 
by the appearance of an air bubble in the urinary bladder or vagina, usually air-free, commonly in proximity to a diseased bowel loop [11].

The sensitivity of CT for the diagnosis of fistulas has been reported in a systematic review. This showed that, based on the pooled results of five studies with surgery and endoscopy as reference standard, the sensitivity was $70 \%$ and specificity 97\% [10]. Pooled results of four studies with an adequate reference standard (endoscopy and/or surgery) showed a sensitivity of MRI for the diagnosis of fistulas of $76 \%$ and specificity of $96 \%$ [10].

\section{Abscesses}

Abscesses are usually located within the leaves of the mesentery or in the retroperitoneum, often being connected to inflamed bowel by a sinus tract [18]. CT has been reported to determine the exact location and extent of an abscess with great reliability, making it the most useful imaging modality to detect intra-abdominal abscesses in CD (Fig. 3A). One study showed that for the detection of intra-abdominal abscesses the sensitivity, specificity, and accuracy of CT compared with surgical findings were $85.7 \%, 87.5 \%$, and $87.2 \%$, respectively [30]. In another prospective study, intraabdominal abscesses were found intraoperatively in 22 patients; the sensitivity of CT for the diagnosis of abscesses was $85 \%$ and
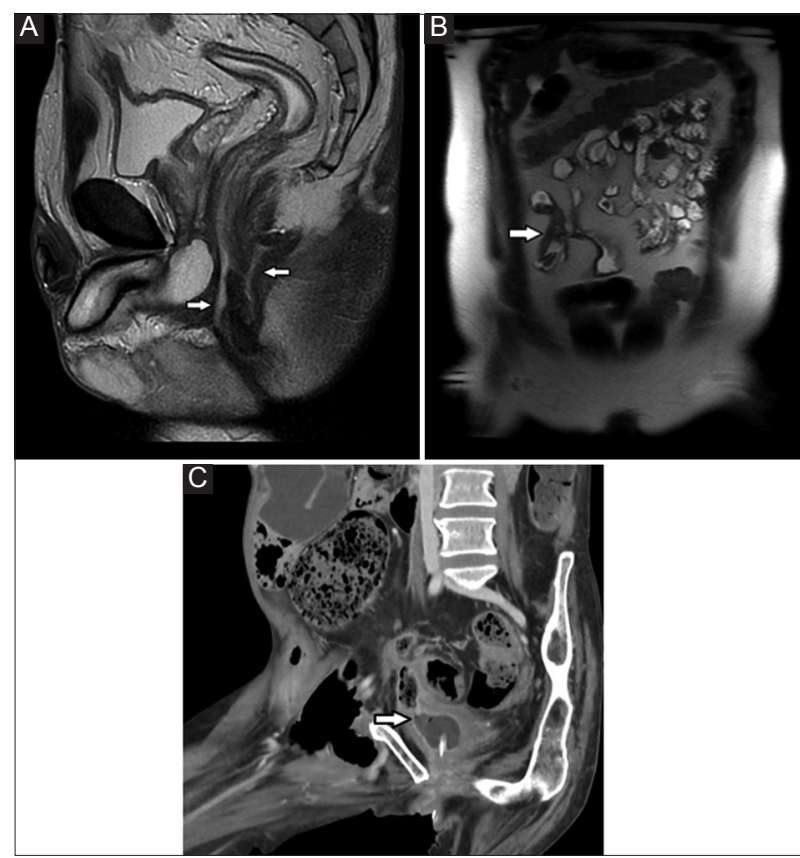

Figure 2 (A) Sagittal T2-weighted single-shot fast spin-echoic image of the pelvis shows complex perianal sepsis with two fistulous tracts arising from the anterior and posterior aspect of the anal canal (arrows); (B) Coronal T2-weighted single-shot fast spin-echo image of the abdomen shows two adjacent small bowel loops tethered to each other, indicative of an enteroenteric fistula (arrow), known as the "starsign"; (C) Coronal computed tomography enterographic section shows a fistula between an inflamed segment of small bowel and the urinary bladder (arrow) specificity was 95\% [31]. Pooled results from 3 studies, in which surgical findings were used as a reference standard to assess the accuracy of MRI in the detection of abscesses, demonstrated a sensitivity of $86 \%$ and a specificity of $93 \%$ [32-34].

\section{Stenotic lesions}

Fibrostenotic disease (Fig. 3B) develops in $18-27 \%$ of patients with CD within 10-20 years of diagnosis [25,35]. Stenosis is usually defined as thickening of the bowel wall with subsequent narrowing of the SB lumen $[1,30]$. Depending on the degree of luminal narrowing caused by the stricture, proximal bowel dilatation may be present. In 2 studies comparing CT with endoscopy and surgery, the sensitivity of CT for the detection of stenosis was $85 \%$ and $90 \%$, while the specificity was $100 \%$ in both [32].

Direct comparison of CT and MRI for the diagnosis of stenosis in a study with 44 patients showed a similar sensitivity ( $85 \%$ vs. $92 \%)$ and specificity (100\% vs. $90 \%$ ) [36]. Pooled results of seven studies with adequate reference standard (endoscopy and/or surgery), demonstrated that the sensitivity of MRI for the diagnosis of stenosis was $89 \%$ and the specificity was $94 \%$ [10]. Better distension was achieved with MR enteroclysis than with MRE, resulting in a higher sensitivity ( $100 \%$ vs. $86 \%$, respectively) and specificity (100\% vs. $93 \%$ ) for detecting stenosis, though the difference was not statistically significant [37]. When CT or MRI is performed before CE, between $27 \%$ and $40 \%$ of patients are excluded from CE by the identification of a stenosis [38,39].

Contrast-enhanced images of the affected segment demonstrate less prominent enhancement compared with that seen on contrast-enhanced images depicting active inflammation. The enhancement seen with fibrostenotic disease is usually restricted to the mucosa. There is no vasa recta engorgement or reactive lymphadenopathy. Unrestricted diffusion is typically seen on diffusionweighted images. However, lower apparent diffusion coefficient values have been associated with the degree of fibrosis, with median apparent diffusion coefficient values for fibrotic strictures being lower than those for non-fibrotic strictures $[40,41]$. Other extraenteric complications of CD identified on cross-sectional imaging include sacroiliitis,
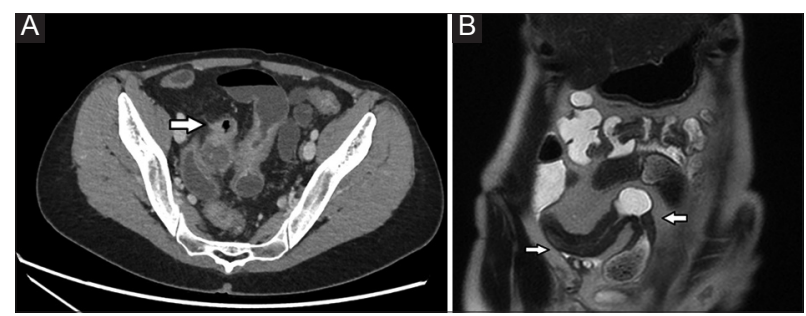

Figure 3 (A) Axial computed tomography enterogram section shows a thick walled gas and fluid collection (arrow) in the pelvis adjacent to inflamed loops of distal ileum; (B) Coronal T2-weighted singleshot fast spin-echo image of the abdomen shows wall thickening and luminal narrowing of two adjacent segments of distal ileum (arrows) with hypertrophy of the surrounding fat - the latter is suggestive of long-standing inflammatory bowel disease with fibrostenotic disease 
renal stones, cholelithiasis, primary sclerosing cholangitis, and lymphoma.

\section{Choice of cross-sectional imaging modality}

\section{Enterography vs. enteroclysis}

The choice of enterography over enteroclysis is pragmatic. CT and MR enteroclysis probably hold a slight advantage over enterography in the detection of early disease, but more established disease is clearly identified with enterography [17]. Enterography eliminates the technical and logistical difficulties of nasojejunal tube insertion, and it eliminates a potential barrier to patient compliance with future examinations [42]. Although jejunal examination is frequently suboptimal, the ileum, the most common site of SB involvement in $\mathrm{CD}$, is usually well demonstrated using enterography techniques [43].

\section{CTE vs. MRE}

The main advantage of MR over CT is the absence of ionizing radiation. Thus MRE is preferentially being utilized for children and young adults who require repeated imaging because of the chronicity of the disease. Other advantages include the ability to obtain a dynamic assessment of the bowel with real-time imaging sequences. Furthermore, MRE also gives an excellent depiction of extraluminal complications of $\mathrm{CD}$, as does CTE [17].

$\mathrm{CT}$ is more appropriate in uncooperative patients, because it requires fewer breath holds than MR imaging, thus improving patient compliance. Moreover, CT should be preferred in the emergency setting, because it depicts perforation more accurately and rapidly, and may also reveal the presence of extraintestinal lesions that may be the cause of an acute abdomen [44].

Other advantages of CTE over MRE include its short imaging time and spatial resolution. It allows multiplanar reformation with isovoxel resolution [17] and also guides interventional procedures, such as percutaneous drainage of intra-abdominal abscesses [45]. CT also has a significantly shorter acquisition time, this being 8-10 sec for CTE and 2030 min for MRE [11].

Limitations of both CTE and MRE include the recognition of fibrotic lesions. While the previously mentioned CTE findings of bowel wall inflammation correlate well with tissue inflammation [46], there is no strong correlation between the radiologic findings presumed to be suggestive of fibrosis and the presence of histological fibrosis. Histological inflammation and fibrosis are strongly correlated with one another, and do not appear to be separable entities [46]. It is in fact likely that CTE and MRE cannot reliably assess the presence of fibrosis with or without associated inflammation, because these two conditions do not exist as mutually exclusive stricture types [46-48].

\section{US vs. MRE}

Both US and MRE are ionizing radiation-free imaging modalities. A comparison of US and MRE in CD patients demonstrated a similar sensitivity, specificity, positive predictive value, and negative predictive value for $C D$ diagnosis. The concordance in terms of CD location between the 2 procedures was high $(\mathrm{k}=0.81)$. However, US was less accurate than MRE in defining CD extension. Moreover, MRI showed a fair concordance with US regarding the identification of strictures $(\mathrm{k}=0.82)$ and abscesses $(\mathrm{k}=0.88)$, but better detection of enteroenteric fistulas $(\mathrm{k}=0.67)$ [49].

Calabrese et al (2016) demonstrated a sensitivity of $89 \%$ and a specificity of $94.3 \%$ for US in the assessment of patients with known $\mathrm{CD}$, with lower accuracy for detecting proximal SB lesions. The administration of an oral contrast agent improved the sensitivity and specificity in determining CD lesions [50].

Panes et al showed that MRI had a high diagnostic accuracy for the diagnosis of suspected CD and for evaluation of disease extension and activity (sensitivity 93\%, specificity 90\%), and was less dependent on the examiner and disease location than US [6].

\section{Radiological scores}

There are currently several MRI-based scoring systems that have been developed in comparison to an external reference standard. The Magnetic Resonance Index of Activity (MaRIA) score can assess both SB and colonic segments [51]. The global MaRIA score is calculated as the sum of the segmental MaRIA scores in the ileum, ascending colon, transverse colon, descending colon, sigmoid colon and rectum (Table 2). In the validation study, MRI features of active inflammation were correlated with the CD Endoscopic Index of Severity (CDEIS). Independent predictors for segmental CDEIS were wall thickness $(\mathrm{P}=0.007)$, relative contrast enhancement $(\mathrm{P}=0.01)$, presence of edema $(\mathrm{P}=0.02)$ and presence of ulcers on MRI $(\mathrm{P}=0.003)$.

The segmental MaRIA score correlates well with the segmental CDEIS $(r=0.82, \mathrm{P}<0.001)$. The overall MaRIA score also correlates with the total CDEIS $(r=0.78)$, CRP levels $(r=0.53)$ and Harvey Bradshaw index $(r=0.56)$. A segmental MaRIA score $\geq 11$ defines severe inflammatory lesions (accuracy 96\%) while a MaRIA score $<7$ detects segmental mucosal healing with reasonable accuracy (83\%), sensitivity

Table 2 Radiological scores

\begin{tabular}{ll} 
Score & Formula \\
\hline MaRIA & $\begin{array}{l}1.5 \times \text { wall thickness }(\mathrm{mm}) \\
+0.02 \times \mathrm{RCE}+5 \times \text { edema }+10 \times \text { ulceration }\end{array}$ \\
& $\begin{array}{l}1.79+1.34 \text { mural thickness }+0.94 \text { mural T2 score. } \\
\text { CDMI }\end{array}$ \\
MEGS & \begin{tabular}{l} 
length -0.192 \\
\hline
\end{tabular}
\end{tabular}

RCE, relative contrast enhancement; MaRIA, magnetic resonance index of activity; CDMI, Crohn's disease MRI index; MEGS, magnetic resonance enterography global score 
(85\%), and specificity (78\%) [51]. It has also been demonstrated that the score correlates well with ulcer healing when MRE is compared to ileocolonoscopy [51]. Potential limitations of the MaRIA score are its failure to take into account the overall length of inflamed segments [51] and its interobserver reproducibility. However, interobserver reproducibility in evaluating inflammation can be improved by modification with DWI [52].

The CD MRI index (CDMI) score (Table 2) was developed through the correlation of MRE findings with transmural histopathology at the time of elective SB surgical resection in CD patients. A simplified model was developed using the MRE parameters that best predicted transmural inflammation [53].

The MRE global score (Table 2) was developed as a modified CDMI to include disease length in order to better capture the full disease burden in CD patients. It was correlated with multiple clinical indices, such as the Harvey Bradshaw index, fecal calprotectin, CRP, and CD activity score [54].

The Nancy Score uses 6 different radiological signs, each recorded in 5 different colonic segments and the ileum, with the total MR score being calculated as the sum of the segmental scores [55]. The Clermont index is similar to the MaRIA score, but includes functional imaging, namely DWI [56]. Another scoring system is the London index. However, when the Clermont index and the London index score were compared to the MaRIA index, the latter had the best operational characteristics for both detecting disease activity and grading severity $[57,58]$. The creation of similar validated CT scoring systems for CD is in progress.

\section{CE}

$\mathrm{CE}$ is a noninvasive procedure and allows a direct and detailed evaluation of the SB mucosa with detection of the earliest lesions of CD [58-61].

\section{Mucosal lesions}

The mucosal lesions are similar to those seen during EGD and ileocolonoscopy. It should be emphasized that ulcerations (Fig. 4A) detected by $\mathrm{CE}$ are not specific for CD [62]. The differential diagnosis includes non-steroidal anti-inflammatory drugs (NSAIDs), lymphoma, radiation enteritis, opportunistic infection in the setting of the human immunodeficiency virus, intestinal tuberculosis, and healthy people [62-64]. It is generally recommended that NSAIDs be stopped four weeks prior to $\mathrm{CE}$; however, the precise period is unknown $[61,65]$. The presence of more than three ulcerations in patients not using NSAIDs should raise the suspicion of CD [66]. Biopsy of the lesions by means of device-assisted enteroscopy can then provide the histological diagnosis. Goldstein et al demonstrated that $11 \%$ of healthy volunteers, not users of NSAIDs, had SB mucosal breaks, and 7\% of patients with a normal baseline CE developed 1-3 mucosal breaks after placebo treatment [63].

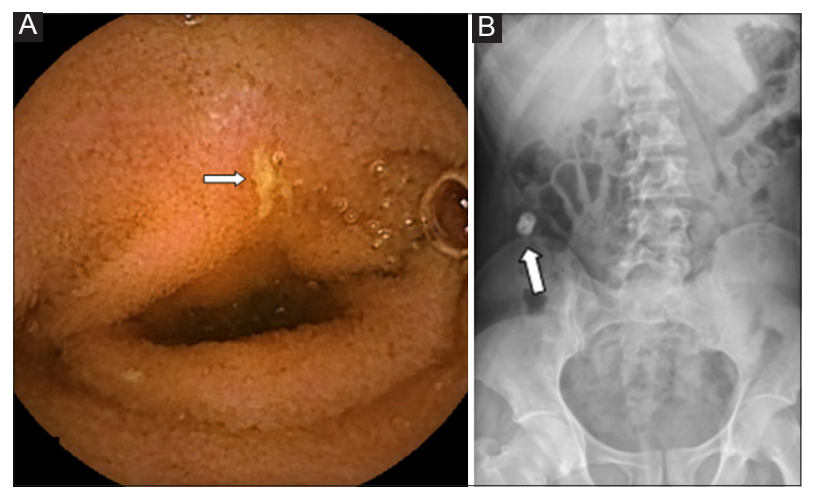

Figure 4 (A) Small bowel ulcer (arrow) noted on capsule endoscopy with Crohn's disease being confirmed on biopsies obtained during enteroscopy; (B) Abdominal radiograph demonstrating a retained patency capsule (arrow)

\section{CE vs. cross-sectional imaging}

In a meta-analysis, the diagnostic yield of $\mathrm{CE}$ in patients with suspected CD was superior to that of SB radiography (52\% vs. $16 \%, \mathrm{P}<0.0001)$ and $\mathrm{CTE}(68 \%$ vs. $21 \%, \mathrm{P}<0.0001)$ [67]. Compared to MRE, CE had a higher diagnostic yield, but the difference did not reach statistical significance (55\% vs. $45 \%$, $\mathrm{P}=0.43)$.

Comparing the diagnostic yield of $\mathrm{CE}$ in patients with known $\mathrm{CD}$, $\mathrm{CE}$ was superior to $\mathrm{SB}$ radiography (71\% vs. $36 \%, \mathrm{P}<0.00001)$ and CTE (71\% vs. $39 \%, \mathrm{P}<0.0001)$ with MRE being equal to $\mathrm{CE}(\mathrm{P}=0.65)$. It should be noted that the studies excluded patients with a radiological suspicion of SB stenosis [67]. In another systematic review, the diagnostic yield of CE was similar to that of MR and US, apart from proximal $\mathrm{SB}$ involvement, for which CE seems to be superior (odds ratio 2.62; $\mathrm{P}=0.03$ ) [68].

Studies have demonstrated that proximal SB involvement is associated with a higher risk of surgery and that jejunal lesions are independent risk factors for future relapse $(\mathrm{P}=0.02)$. This superior accuracy may therefore have prognostic value [69].

\section{Assessment of disease activity}

Currently there are two validated indices (Tables 3 and 4): the Lewis score and the CE CD Activity Index (CECDAI). There is a good correlation between the two, $\mathrm{P}<0.0001[70,71]$. For the Lewis score, a level of $<135$ suggests normal SB or clinically insignificant inflammation; 135-790 means mild disease activity and $>790$ indicates moderate to severe disease activity [70]. CECDAI levels of 3.8 and 5.8 corresponded to Lewis scores of 135 and 790, respectively [72,73].

\section{CE and inflammatory markers}

Data from small, prospective cohorts suggest that the diagnostic yield of $\mathrm{CE}$ is highest in patients with suggestive 
Table 3 Lewis score

\begin{tabular}{llll}
\hline Parameter & Weightings (calculated for each tertile) & & \\
\hline Villous appearance & Appearance & Longitudinal extent & Descriptors \\
\hline & $0=$ Normal & $8=$ Short segment & $1=$ Single \\
& $1=$ Edematous & $12=$ Long segment & $14=$ Patchy \\
& & $20=$ Whole tertile & $17=$ Diffuse \\
\hline Ulcer & Number & Longitudinal extent & Descriptors \\
\hline & $0=$ None & $5=$ Short segment & $9=$ Less than $25 \%$ of circumference \\
& $3=$ Single & $10=$ Long segment & $12=25 \%$ to $50 \%$ of circumference \\
& $5=$ Few & $25=$ Whole tertile & $18=$ Greater than $50 \%$ of circumference \\
& $10=$ Multiple & & \\
\hline Parameter & Weightings (rated for the whole study) & & \\
\hline Stenosis & Number & Appearance & Passage of capsule past stricture \\
\hline & $0=$ None & $24=$ Ulcerated & $7=$ Traversed \\
& $14=$ Single & $2=$ Non-ulcerated & $10=$ Not traversed
\end{tabular}

Short segment: $\leq 10 \%$ of the tertile; Long segment: $11 \%-50 \%$ of a tertile; Whole tertile: $\geq 50 \%$ of the tertile; Few: two to seven lesions; Multiple: eight or more ulcers, two or more stenoses. Gralnek et al [74]

Table 4 Capsule endoscopy Crohn's disease activity index

\begin{tabular}{llll}
\hline A: Inflammation & B: Extent & C: Stricturing & Score for each segment \\
\hline $0=$ None & $0=$ None & $0=$ None & A + C \\
$1=$ Mild to moderate edema/hyperemia/denudation & $1=$ Focal & $1=$ Single (passed) \\
$2=$ Severe edema/hyperemia/denudation & $2=$ Patchy & $2=$ Multiple (passed) \\
$3=$ Small ulcer $(5 \mathrm{~mm})$ & $3=$ Diffuse & $3=$ Obstructing \\
$4=$ Moderate ulcer $(5-20 \mathrm{~mm})$ & & \\
$5=$ Large ulcer $(20 \mathrm{~mm})$ & & \\
\hline Gal et al. $[71]$ & &
\end{tabular}

CD symptoms and elevated inflammatory markers [74,75], although this finding could not be replicated in larger retrospective cohorts $[72,76]$. Patients with suspected CD who have calprotectin levels $<50 \mu \mathrm{g} / \mathrm{g}$ have a very low likelihood of positive CE diagnosis (negative predictive value: -91.8\%) [77].

\section{Contraindications for CE}

Contraindications for CE include gastrointestinal obstruction, strictures and swallowing disorders, although the latter can be overcome by direct endoscopic placement $[78,79]$. In a comprehensive literature review, capsule retention occurred in $1.5 \%$ of patients examined for suspected CD $[80,81]$ and $2-13 \%$ of patients with established CD [82].

If $\mathrm{SB}$ stenosis is suspected, $\mathrm{CE}$ should be preceded by either cross-sectional imaging or a patency capsule
(Fig. 4B) so as to avoid capsule retention [65,71]. In a study by Yadav et al, the patency capsule and non-enteroclysis radiologic examinations were equally reliable for excluding SB obstruction or strictures. The advantage of radiologic tests is that they do not depend on intestinal motility, as does the patency capsule. On the other hand, they may overestimate the risk of functional obstruction [83].

\section{Enteroscopy}

Traditionally, endoscopic examination of the SB has been challenging [84], though technological advances have extended the reach of the endoscopist, allowing for better access. Enteroscopy has a role in the management of both suspected as well as established CD [64, 84], allowing for histological sampling in areas not accessible with the standard EGD and 
ileocolonoscopy [85]. Its other role is in the form of therapeutic interventions, which include pneumatic dilatation, injection of corticosteroid and immunomodulatory drugs and stent insertion $[86,87]$. Such therapeutic strategies have resulted in clinical, biochemical and endoscopic improvement in patients with CD $[88,89]$.

Pneumatic dilation is the most common therapeutic use of enteroscopy in $\mathrm{CD}$, and is a valuable alternative to surgical intervention. In a systematic review of 13 retrospective studies published over a 17-year period, Hassan et al demonstrated an $86 \%$ technical success rate of pneumatic dilatation in the treatment of strictures due to CD [90]. Long-term clinical efficacy was also evident, as $58 \%$ of patients did not require surgery over the follow-up period, a mean of 33 months [90].

Balloon dilatation is indicated for the treatment of noninflammatory SB strictures of up to $4-6 \mathrm{~cm}$ in length [91]. Efficacy is significantly lower in strictures longer than $4 \mathrm{~cm}$ [88], and pneumatic dilatation of longer and/or inflammatory strictures has been associated with a significantly increased risk of perforation [91]. Enteroscopy also allows for the retrieval of retained capsules used in wireless CE [91].

\section{Push-and-pull enteroscopy}

Push-and-pull enteroscopy is the oldest type of enteroscopy [84]. Its clinical utility and influence on management have been demonstrated in small studies $[92,93]$. In a study by Darbari et al, push-and-pull enteroscopy confirmed the presence of proximal SB disease in $84 \%$ of children with a clinical suspicion of SB pathology [94]. Clinical management was affected in $77 \%$ of the pediatric population studied.

\section{Device-assisted enteroscopy (DAE)}

DAE is a newer method that allows for the endoluminal examination of the SB by any endoscopic technique that includes assisted progression [84]. The 3 main DAE techniques are double-balloon enteroscopy (DBE), single-balloon enteroscopy (SBE), and spiral enteroscopy (SE).

\section{Balloon-assisted endoscopy (BAE)}

BAE involves the stepwise progression of a scope through the small intestine with a balloon-loaded overtube used as a straightening device [84]. The addition of an inflatable balloon at the distal end of the overtube allows for better mucosal grip and stabilizes its position within the intestinal lumen. DBE makes use of a second balloon attached to the tip of the endoscope. If required, complete intubation of the SB is possible, typically through a combined approach through the mouth and the anus [95].

The diagnostic yield of DBE has been shown to influence the management of CD patients $[88,89,96]$. In a prospective study by Mensink et al, $70 \%$ of patients with a clinical suspicion of SB
CD were confirmed to have SB CD using this technique [89]. Half of these lesions were inaccessible by conventional endoscopy. The diagnosis achieved with this technique led to a change in therapy in $74 \%$ of patients, with a resultant clinical and endoscopic remission in $88 \%$ at 1 -year follow up [89].

SBE follows the same principles as DBE, except that the tip of the scope is used as an anchor, rather than a second balloon [84,97]. A randomized international multicenter study by Domagk et al was set up to compare the insertion depth of the two techniques. The study revealed a mean insertion depth of $253 \mathrm{~cm}$ (DBE) and $258 \mathrm{~cm}$ (SBE) with anterograde procedures and a mean insertion depth of $107 \mathrm{~cm}$ (DBE) and $118 \mathrm{~cm}$ (SBE) with retrograde procedures [97].

Whereas complete visualization of the SB is more likely with DBE ( $18 \%$ vs. $11 \%$ of procedures), the diagnostic yield in patients with SB pathology is comparable with both techniques [97]. In a meta-analysis comparing both techniques, $\mathrm{DBE}$ was superior to $\mathrm{SBE}$ for $\mathrm{SB}$ visualization $(\mathrm{P}=0.004)$, but had similar rates of providing a diagnosis $(\mathrm{P}=0.62)$, therapeutic yield $(\mathrm{P}=0.09)$ and complication rate $(\mathrm{P}=0.9)$ [98]. Data are limited with regards to comparative studies in $\mathrm{CD}$.

\section{Spiral Enteroscopy (SE)}

SE is a newer technique that employs a spiral tipped overtube. This travels ahead of the enteroscope, telescoping the SB and allowing visualization of the mucosa [84]. Likewise, one can insert it both antero- and retrogradely. A recent metaanalysis revealed that the maximum depth of insertion and diagnostic yield is comparable to that of BAE [98], though further comparative studies are required specifically for CD.

\section{Complications}

The use of push enteroscopy and diagnostic DAE in the investigation of $\mathrm{CD}$ has a complication rate of less than $1 \%$, the main complication being post-procedural abdominal pain, hyperamylasemia and pancreatitis $(0.3 \%)$ if the oral route is used $[99,100]$. On the other hand, complication rates of therapeutic DAE are higher in CD [101], Gustavsson et al published the largest study to date on this matter and reported an overall complication rate of 5.3\%, though rates were as high as $9.3 \%$ with the largest dilatation diameter $(25 \mathrm{~mm})$ [101].

\section{Concluding remarks}

Small-bowel follow through has been superseded by newer radiological modalities (contrast-enhanced US, MRI, and CT) as well as endoscopic modalities (CE and enteroscopy) in the investigation and management of $\mathrm{CD}$ patients. A range of factors determine the choice of modality for SB assessment. These include local availability and expertise, acute vs. nonacute clinical scenarios, patient preference and cooperation during the test. US and MRE should be preferred to CTE 
because of the absence of ionizing radiation, especially in younger patients. CE should be used with caution when clinical symptoms indicate obstructive or stricturing $\mathrm{CD}$, especially if not preceded by a patency capsule. Enteroscopy allows for histological sampling and therapeutic intervention.

\section{References}

1. Gomollón F, Dignass A, Annese V, et al; ECCO. $3^{\text {rd }}$ European Evidence-based Consensus on the Diagnosis and Management of Crohn's Disease 2016: Part 1: diagnosis and medical management. J Crohns Colitis 2017;11:3-25.

2. Samuel S, Bruining DH, Loftus EV Jr, et al. Endoscopic skipping of the distal terminal ileum in Crohn's disease can lead to negative results from ileocolonoscopy. Clin Gastroenterol Hepatol 2012;10:1253-1259.

3. Berliner L, Redmond P, Purow E, Megna D, Sottile V. Computed tomography in Crohn's disease. Am J Gastroenterol 1982;77:548-553.

4. Kuehle C, Ajaj W, Ladd S, Massing S, Barkhausen J, Lauenstein TC. Hydro-MRI of the small bowel: effect of contrast volume, timing of contrast administration, and data acquisition on bowel distention. AJR Am J Roentgenol 2006;187:W375-W385.

5. Huprich JE, Fletcher JG. CT enterography: principles, technique and utility in Crohn's disease. Eur J Radiol 2009;69:393-397.

6. Panes J, Bouhnik Y, Reinisch W, et al. Imaging techniques for assessment of inflammatory bowel disease: joint ECCO and ESGAR evidence-based consensus guidelines. J Crohns Colitis 2013;7:556-585.

7. Baker ME, Hara AK, Platt JF, Maglinte DD, Fletcher JG. CT enterography for Crohn's disease: optimal technique and imaging issues. Abdom Imaging 2015;40:938-952.

8. Bruining DH, Siddiki HA, Fletcher JG, et al. Benefit of computed tomography enterography in Crohn's disease: effects on patient management and physician level of confidence. Inflamm Bowel Dis 2012;18:219-225.

9. Haas K, Rubesova E, Bass D. Role of imaging in the evaluation of inflammatory bowel disease: how much is too much? World $J$ Radiol 2016;8:124-131.

10. Panés J, Bouzas R, Chaparro M, et al. Systematic review: the use of ultrasonography, computed tomography and magnetic resonance imaging for the diagnosis, assessment of activity and abdominal complications of Crohn's disease. Aliment Pharmacol Ther 2011;34:125-145.

11. Amitai MM, Ben-Horin S, Eliakim R, Kopylov U. Magnetic resonance enterography in Crohn's disease: a guide to common imaging manifestations for the IBD physician. J Crohns Colitis 2013;7:603-615.

12. Seo N, Park SH, Kim KJ, et al. MR Enterography for the evaluation of small-bowel inflammation in Crohn disease by using diffusion weighted imaging without intravenous contrast material: a prospective noninferiority study. Radiology 2016;278:762-772.

13. Messaris E, Chandolias N, Grand D, Pricolo V. Role of magnetic resonance enterography in the management of Crohn disease. Arch Surg 2010;145:471-475.

14. Mendoza JL, González-Lama Y, Taxonera C, et al. Using of magnetic resonance enterography in the management of Crohn's disease of the small intestine: first year of experience. Rev Esp Enferm Dig 2012;104:578-583.

15. Kucharzik T, Kannengiesser K, Petersen F. The use of ultrasound in inflammatory bowel disease. Ann Gastroenterol 2017;30:135-144.

16. Quaia E. Contrast-enhanced ultrasound of the small bowel in Crohn's disease. Abdom Imaging 2013;38:1005-1013.
17. Tolan DJ, Greenhalgh R, Zealley IA, Halligan S, Taylor SA. MR enterographic manifestations of small bowel Crohn disease. Radiographics 2010;30:367-384.

18. Paulsen SR, Huprich JE, Fletcher JG, et al. CT enterography as a diagnostic tool in evaluating small bowel disorders: review of clinical experience with over 700 cases. Radiographics 2006;26:641-657.

19. Booya F, Fletcher JG, Johnson CD, et al. CT enterography: detection of active Crohn's disease during optimal bowel wall enhancement. Radiological Society of North America scientific assembly and annual meeting program: Radiological Society of North America 2004;611.

20. Spalinger J, Patriquin H, Miron MC, et al. Doppler US in patients with crohn disease: vessel density in the diseased bowel reflects disease activity. Radiology 2000;217:787-791.

21. Haber HP, Busch A, Ziebach R, Dette S, Ruck P, Stern M. Ultrasonographic findings correspond to clinical, endoscopic, and histologic findings in inflammatory bowel disease and other enterocolitides. J Ultrasound Med 2002;21:375-382.

22. Neye H, Voderholzer W, Rickes S, Weber J, Wermke W, Lochs H. Evaluation of criteria for the activity of Crohn's disease by power Doppler sonography. Dig Dis 2004;22:67-72.

23. Muradali D, Goldberg DR. US of gastrointestinal tract disease. Radiographics 2015;35:50-68.

24. Lo Re G, Cappello M, Tudisca C, et al. CT enterography as a powerful tool for the evaluation of inflammatory activity in Crohn's disease: relationship of CT findings with CDAI and acute-phase reactants. Radiol Med 2014;119:658-666.

25. Lee SS, Ha HK, Yang SK, et al. CT of prominent pericolic or perienteric vasculature in patients with Crohn's disease: correlation with clinical disease activity and findings on barium studies. $A J R$ Am J Roentgenol 2002;179:1029-1036.

26. Sakurai T, Katsuno T, Saito K, et al. Mesenteric findings of CT enterography are well correlated with the endoscopic severity of Crohn's disease. Eur J Radiol 2017;89:242-248.

27. Hyun SB, Kitazume Y, Nagahori M, et al. Magnetic resonance enterocolonography is useful for simultaneous evaluation of small and large intestinal lesions in Crohn's disease. Inflamm Bowel Dis 2011;17:1063-1072.

28. Qiu Y, Mao R, Chen BL, et al. Systematic review with meta-analysis: magnetic resonance enterography vs. computed tomography enterography for evaluating disease activity in small bowel Crohn's disease. Aliment Pharmacol Ther 2014;40:134-146.

29. Paquet N, Glickman JN, Erturk SM, Ros PR, Heverhagen JT, Patak MA. Crohn's disease activity: abdominal computed tomography histopathology correlation. Eur J Radiol Open 2016;3:74-78.

30. Maconi G, Sampietro GM, Parente F, et al. Contrast radiology, computed tomography and ultrasonography in detecting internal fistulas and intra-abdominal abscesses in Crohn's disease: a prospective comparative study. Am J Gastroenterol 2003;98:1545-1555.

31. Choi D, Jin Lee S, Ah Cho Y, et al. Bowel wall thickening in patients with Crohn's disease: CT patterns and correlation with inflammatory activity. Clin Radiol 2003;58:68-74.

32. Lee SS, Kim AY, Yang SK, et al. Crohn disease of the small bowel: comparison of CT enterography, MR enterography, and small-bowel follow-through as diagnostic techniques. Radiology 2009;251:751-761.

33. Zappa M, Stefanescu C, Cazals-Hatem D, et al. Which magnetic resonance imaging findings accurately evaluate inflammation in small bowel Crohn's disease? A retrospective comparison with surgical pathologic analysis. Inflamm Bowel Dis 2011;17:984-993.

34. Martínez MJ, Ripollés T, Paredes JM, Blanc E, Martí-Bonmatí L. Assessment of the extension and the inflammatory activity in Crohn's disease: comparison of ultrasound and MRI. Abdom 
Imaging 2009;34:141-148.

35. Cosnes J, Cattan S, Blain A, et al. Long-term evolution of disease behavior of Crohn's disease. Inflamm Bowel Dis 2002;8:244-250.

36. Fiorino G, Bonifacio C, Peyrin-Biroulet L, et al. Prospective comparison of computed tomography enterography and magnetic resonance enterography for assessment of disease activity and complications in ileocolonic Crohn's disease. Inflamm Bowel Dis 2011;17:1073-1080.

37. Negaard A, Paulsen V, Sandvik L, et al. A prospective randomized comparison between two MRI studies of the small bowel in Crohn's disease, the oral contrast method and MR enteroclysis. Eur Radiol 2007;17:2294-2301.

38. Voderholzer WA, Beinhoelzl J, Rogalla P, et al. Small bowel involvement in Crohn's disease: a prospective comparison of wireless capsule endoscopy and computed tomography enteroclysis. Gut 2005,54:369-373.

39. Wiarda BM, Mensink PB, Heine DG, et al. Small bowel Crohn's disease: MR enteroclysis and capsule endoscopy compared to balloon-assisted enteroscopy. Abdom Imaging 2012;37:397-403.

40. Tielbeek JA, Makanyanga JC, Bipat S, et al. Grading Crohn disease activity with MRI: interobserver variability of MRI features, MRI scoring of severity, and correlation with Crohn disease endoscopic index of severity. AJR Am J Roentgenol 2013;201:1220-1228.

41. Kaushal P, Somwaru AS, Charabaty A, Levy AD. MR enterography of inflammatory bowel disease with endoscopic correlation. Radiographics 2017;37:116-131.

42. Negaard A, Sandvik L, Berstad AE, et al. MRI of the small bowel with oral contrast or nasojejunal intubation in Crohn's disease: randomized comparison of patient acceptance. Scand $J$ Gastroenterol 2008;43:44-51.

43. Masselli G, Casciani E, Polettini E, Gualdi G. Comparison of MR enteroclysis with MR enterography and conventional enteroclysis in patients with Crohn's disease. Eur Radiol 2008;18:438-447.

44. Masselli G, Gualdi G. MR imaging of the small bowel. Radiology 2012;264:333-348

45. Rypens F, Dubois J, Garel L, Deslandres C, Saint-Vil D. Percutaneous drainage of abdominal abscesses in pediatric Crohn's disease. AJR Am J Roentgenol 2007;188:579-585

46. Adler J, Punglia DR, Dillman JR, et al. Computed tomography enterography findings correlate with tissue inflammation, not fibrosis in resected small bowel Crohn's disease. Inflamm Bowel Dis 2012; 18:849-856.

47. Barkmeier D, Dillman J, Al-Hawary M. MR enterographyhistology comparison in resected pediatric small bowel Crohn disease strictures: can imaging predict fibrosis? Pediatr Radiol 2016;46:498-507.

48. Rieder F, Latella G, Magro F, et al. European Crohn's and Colitis Organisation Topical Review on Prediction, Diagnosis and Management of Fibrostenosing Crohn's Disease. J Crohns Colitis 2016;10:873-885.

49. Castiglione F, Mainenti PP, De Palma GD, et al. Noninvasive diagnosis of small bowel Crohn's disease: direct comparison of bowel sonography and magnetic resonance enterography. Inflamm Bowel Dis 2013;19:991-998.

50. Calabrese E, Maaser C, Zorzi F, et al. Bowel Ultrasonography in the Management of Crohn's Disease. A Review with Recommendations of an International Panel of Experts. Inflamm Bowel Dis 2016;22:1168-1183.

51. Ordás I, Rimola J, Rodríguez S, et al. Accuracy of magnetic resonance enterography in assessing response to therapy and mucosal healing in patients with Crohn's disease. Gastroenterology 2014;146:374-382.e1.

52. Kim JS, Jang HY, Park SH, et al. MR enterography assessment of bowel inflammation severity in Crohn disease using the MR index of activity score: modifying roles of DWI and effects of contrast phases. AJR Am J Roentgenol 2017;208:1022-1029.
53. Steward MJ, Punwani S, Proctor I, et al. Non-perforating small bowel Crohn's disease assessed by MRI enterography: derivation and histopathological validation of an MR-based activity index. Eur J Radiol 2012;81:2080-2088.

54. Makanyanga JC, Pendsé D, Dikaios N, et al. Evaluation of Crohn's disease activity: initial validation of a magnetic resonance enterography global score (MEGS) against faecal calprotectin. Eur Radiol 2014;24:277-287.

55. Oussalah A, Laurent V, Bruot O, et al. Diffusion-weighted magnetic resonance without bowel preparation for detecting colonic inflammation in inflammatory bowel disease. Gut 2010;59:1056-1065.

56. Buisson A, Pereira B, Goutte M, et al. Magnetic resonance index of activity (MaRIA) and Clermont score are highly and equally effective MRI indices in detecting mucosal healing in Crohn's disease. Dig Liver Dis 2017;49:1211-1217.

57. Rimola J, Alvarez-Cofiño A, Pérez-Jeldres T, et al. Comparison of three magnetic resonance enterography indices for grading activity in Crohn's disease. J Gastroenterol 2017;52:585-593.

58. Koulaouzidis A, Rondonotti E, Karargyris A. Small-bowel capsule endoscopy: a ten-point contemporary review. World J Gastroenterol 2013;19:3726-3746.

59. Jensen MD, Nathan T, Rafaelsen SR, Kjeldsen J. Diagnostic accuracy of capsule endoscopy for small bowel Crohn's disease is superior to that of MR enterography or CT enterography. Clin Gastroenterol Hepatol 2011;9:124-129.

60. Pennazio M, Spada C, Eliakim R, et al. Small-bowel capsule endoscopy and device-assisted enteroscopy for diagnosis and treatment of small-bowel disorders: European Society of Gastrointestinal Endoscopy (ESGE) Clinical Guideline. Endoscopy 2015;47:352-376.

61. Mitselos IV, Christodoulou DK, Katsanos KH, Tsianos EV. Role of wireless capsule endoscopy in the follow-up of inflammatory bowel disease. World J Gastrointest Endosc 2015;7:643-651.

62. Bourreille A, Ignjatovic A, Aabakken L, et al; World Organisation of Digestive Endoscopy (OMED) and the European Crohn's and Colitis Organisation (ECCO). Role of small-bowel endoscopy in the management of patients with inflammatory bowel disease: an international OMED-ECCO consensus. Endoscopy 2009;41:618-637.

63. Goldstein JL, Eisen GM, Lewis B, Gralnek IM, Zlotnick S, Fort JG. Video capsule endoscopy to prospectively assess small bowel injury with celecoxib, naproxen plus omeprazole, and placebo. Clin Gastroenterol Hepatol 2005;3:133-141.

64. Bar-Meir S. Review article: capsule endoscopy - are all small intestinal lesions Crohn's disease? Aliment Pharmacol Ther 2006;24(Suppl 3):19-21.

65. Annese V, Daperno M, Rutter MD, et al; European Crohn's and Colitis Organisation. European evidence based consensus for endoscopy in inflammatory bowel disease. J Crohns Colitis 2013;7:982-1018.

66. Mow WS, Lo SK, Targan SR, et al. Initial experience with wireless capsule enteroscopy in the diagnosis and management of inflammatory bowel disease. Clin Gastroenterol Hepatol 2004;2:31-40.

67. Dionisio PM, Gurudu SR, Leighton JA, et al. Capsule endoscopy has a significantly higher diagnostic yield in patients with suspected and established small-bowel Crohn's disease: a meta-analysis. Am J Gastroenterol 2010;105:1240-1248.

68. Kopylov U, Yung DE, Engel T, et al. Diagnostic yield of capsule endoscopy versus magnetic resonance enterography and small bowel contrast ultrasound in the evaluation of small bowel Crohn's disease: Systematic review and meta-analysis. Dig Liver Dis 2017;49:854-863.

69. Flamant M, Trang C, Maillard O, et al. The prevalence and outcome of jejunal lesions visualized by small bowel capsule endoscopy in 
Crohn's disease. Inflamm Bowel Dis 2013;19:1390-1396.

70. Gralnek IM, Defranchis R, Seidman E, Leighton JA, Legnani P, Lewis BS. Development of a capsule endoscopy scoring index for small bowel mucosal inflammatory change. Aliment Pharmacol Ther 2008;27:146-154.

71. Gal E, Geller A, Fraser G, Levi Z, Niv Y. Assessment and validation of the new capsule endoscopy Crohn's disease activity index (CECDAI). Dig Dis Sci 2008;53:1933-1937.

72. Kopylov U, Yablecovitch D, Lahat A, et al. Detection of small bowel mucosal healing and deep remission in patients with known small bowel Crohn's disease using biomarkers, capsule endoscopy, and imaging. Am J Gastroenterol 2015;110:1316-1323.

73. Koulaouzidis A, Douglas S, Plevris JN. Lewis score correlates more closely with fecal calprotectin than capsule endoscopy Crohn's disease activity index. Dig Dis Sci 2012;57:987-993.

74. Katsinelos P, Fasoulas K, Beltsis A, et al. Diagnostic yield and clinical impact of wireless capsule endoscopy in patients with chronic abdominal pain with or without diarrhea: a Greek multicenter study. Eur J Intern Med 2011;22:e63-e66.

75. De Bona M, Bellumat A, Cian E, Valiante F, Moschini A, De Boni M. Capsule endoscopy findings in patients with suspected Crohn's disease and biochemical markers of inflammation. Dig Liver Dis 2006;38:331-335.

76. Kopylov U, Nemeth A, Koulaouzidis A, et al. Small bowel capsule endoscopy in the management of established Crohn's disease: clinical impact, safety, and correlation with inflammatory biomarkers. Inflamm Bowel Dis 2015;21:93-100.

77. Kopylov U, Yung DE, Engel T, et al. Fecal calprotectin for the prediction of small-bowel Crohn's disease by capsule endoscopy: a systematic review and meta-analysis. Eur J Gastroenterol Hepatol 2016;28:1137-1144.

78. Triester SL, Leighton JA, Leontiadis GI, et al. A meta-analysis of the yield of capsule endoscopy compared to other diagnostic modalities in patients with non-stricturing small bowel Crohn's disease. Am J Gastroenterol 2006;101:954-964.

79. Van Weyenberg SJ, Bouman K, Jacobs MA, et al. Comparison of MR enteroclysis with video capsule endoscopy in the investigation of small-intestinal disease. Abdom Imaging 2013;38:42-51.

80. Nemeth A, Kopylov U, Koulaouzidis A, et al. Use of patency capsule in patients with established Crohn's disease. Endoscopy 2016;48:373-379.

81. Liao Z, Gao R, Xu C, Li ZS. Indications and detection, completion, and retention rates of small-bowel capsule endoscopy: a systematic review. Gastrointest Endosc 2010;71:280-286.

82. Cheifetz AS, Kornbluth AA, Legnani P, et al. The risk of retention of the capsule endoscope in patients with known or suspected Crohn's disease. Am J Gastroenterol 2006;101:2218-2222.

83. Yadav A, Heigh RI, Hara AK, et al. Performance of the patency capsule compared with nonenteroclysis radiologic examinations in patients with known or suspected intestinal strictures. Gastrointest Endosc 2011;74:834-839.

84. Moreels TG. History of endoscopic devices for the exploration of the small bowel. Acta Gastroenterol Belg 2009;72:335-337.

85. Seiderer J, Herrmann K, Diepolder H, et al. Double-balloon enteroscopy versus magnetic resonance enteroclysis in diagnosing suspected small-bowel Crohn's disease: results of a pilot study. Scand J Gastroenterol 2007;42:1376-1385.

86. Pennazio M. Crohn's disease: diagnostic and therapeutic potential of modern small-bowel endoscopy. Gastrointest Endosc 2007;66:S91-S93.

87. Kochhar R, Poornachandra KS. Intralesional steroid injection therapy in the management of resistant gastrointestinal strictures. World J Gastrointest Endosc 2010;2:61-68.

88. Mensink PB, Groenen MJ, van Buuren HR, Kuipers EJ, van der Woude CJ. Double-balloon enteroscopy in Crohn's disease patients suspected of small bowel activity: findings and clinical impact. J Gastroenterol 2009;44:271-276.

89. Mensink PB, Aktas H, Zelinkova Z, West RL, Kuipers EJ, van der Woude CJ. Impact of double-balloon enteroscopy findings on the management of Crohn's disease. Scand J Gastroenterol 2010;45:483-489.

90. Hassan C, Zullo A, De Francesco V, et al. Systematic review: Endoscopic dilatation in Crohn's disease. Aliment Pharmacol Ther 2007;26:1457-1464.

91. Sunada K, Yamamoto H, Yano T, Sugano K. Advances in the diagnosis and treatment of small bowel lesions in Crohn's disease using double-balloon endoscopy. Ther Adv Gastroenterol 2009;2:357-366.

92. Benz C, Jakobs R, Riemann JF. Do we need the overtube for pushenteroscopy? Endoscopy 2001;33:658-661.

93. Harewood GC, Gostout CJ, Farrell MA, Knipschield MA. Prospective controlled assessment of variable stiffness enteroscopy. Gastrointest Endosc 2003;58:267-271.

94. Darbari A, Kalloo AN, Cuffari C. Diagnostic yield, safety, and efficacy of push enteroscopy in pediatrics. Gastrointest Endosc 2006;64:224-228.

95. Mönkemüller K, Fry LC, Bellutti M, Malfertheiner P. Balloonassisted enteroscopy: unifying double-balloon and single-balloon enteroscopy. Endoscopy 2008;40:537.

96. Cazzato IA, Cammarota G, Nista EC, et al. Diagnostic and therapeutic impact of double-balloon enteroscopy (DBE) in a series of 100 patients with suspected small bowel diseases. Dig Liver Dis 2007;39:483-487.

97. Domagk D, Mensink P, Aktas H, et al. Single- vs. double-balloon enteroscopy in small-bowel diagnostics: a randomized multicenter trial. Endoscopy 2011;43:472-476.

98. Wadhwa V, Sethi S, Tewani S, et al. A meta-analysis on efficacy and safety: single-balloon vs. double-balloon enteroscopy. Gastroenterol Rep (Oxf) 2015;3:148-155.

99. Mensink PB, Haringsma J, Kucharzik T, et al. Complications of double balloon enteroscopy: a multicenter survey. Endoscopy 2007;39:613-615.

100.Arulanandan A, Dulai PS, Singh S, Sandborn WJ, Kalmaz D. Systematic review: Safety of balloon assisted enteroscopy in Crohn's disease. World J Gastroenterol 2016;22:8999-9011.

101.Gustavsson A, Magnuson A, Blomberg B, Andersson M, Halfvarson J, Tysk C. Endoscopic dilation is an efficacious and safe treatment of intestinal strictures in Crohn's disease. Aliment Pharmacol Ther 2012;36:151-158. 